\title{
Content-Based Social Network Analysis of Mailing Lists
}

\section{by Angela Bohn, Ingo Feinerer, Kurt Hornik and Patrick Mair}

\begin{abstract}
Social Network Analysis (SNA) provides tools to examine relationships between people. Text Mining (TM) allows capturing the text they produce in Web 2.0 applications, for example, however it neglects their social structure. This paper applies an approach to combine the two methods named "content-based SNA". Using the $\mathrm{R}$ mailing lists, R-help and R-devel, we show how this combination can be used to describe people's interests and to find out if authors who have similar interests actually communicate. We find that the expected positive relationship between sharing interests and communicating gets stronger as the centrality scores of authors in the communication networks increase.
\end{abstract}

\section{Introduction}

Social Network Analysis (SNA) provides powerful methods to study the relationships between people expressed as binary or weighted adjacency matrices. It can be used to find influential or popular nodes, communities and informal hierarchies. However, it is limited in the sense that it cannot capture the context of their encounter. An actor might be a regarded adviser or trend setter concerning one topic and might not know anything about another. If all his or her relationships are expressed as purely numerical networks, a differentiation is not possible.

In open source software communities, a large part of the developers' communication and thus collaboration happens electronically via e-mails and forums. In the $\mathrm{R}$ mailing lists, R-help and R-devel, all kinds of application and development related questions are discussed. From experts to newcomers, everyone shares the same platform, so a view on the entire network does not offer a detailed picture, leaving the question of how well the communication really works in the $\mathrm{R}$ community and which role the most central actors play in this context. As bad coordination can be reflected by redundant code or hardly compatible packages, it is important that developers and users working in the same field stay in contact.

In this paper, we use content-based SNA, an approach to combine SNA and Text Mining (TM) in order to find out to which extent sharing interests is associated with communicating in two R mailing lists. Content-based SNA consists of extracting overlapping topic related subnetworks from the entire communication network. The paper shows how the au- thors' interests can be found based on their centralities in these topic-based subnetworks. By comparing the networks showing who shares interests with their communication networks, we find that communicating is the more associated with sharing interests the more central the authors are in the communication networks. We argue that this finding is due to the motives of authors to use the mailing lists.

\section{Related work}

There are several approaches to combine SNA and TM. One has to distinguish between combinations that enrich TM through SNA and those that enrich SNA through TM. One of the most prominent applications on the TM side is used by the search engines Google and Yahoo. They rank search results found by TM procedures according to centrality measures based on hyperlink graphs (Brin and Page, 1998; Kleinberg, 1999). Another very important TM plus SNA application is the summarization of texts by calculating centrality measures in word networks (networks where nodes represent words that are connected if they appear in the same text unit). The results are often visualized as tag clouds (Erkan and Radev, 2004; Vanderwende et al., 2004).

However, approaches to enrich an SNA with TM are not as numerous. McCallum et al. (2007) introduced the author-recipient-topic model which consists of fitting a multinomial distribution over topics and authors/recipients of a message simultaneously. The results are combinations of concepts and pairs of authors and recipients that characterize the network. By studying the Enron e-mail corpus, they defined social roles based on such combinations. For example the relationships of an Enron lawyer to a few other people were characterized by the concept "legal contracts". A similar approach was applied to authors of Wikipedia articles by Chang et al. (2009).

The tradition this paper stands in is called "content-based SNA". In content-based SNA, the network is partitioned into several overlapping subgraphs of people who discuss the same topic. Examples in the literature comprise Velardi et al. (2008), who analyzed the evolution of content-based communication networks in a company by measuring the agent-aggregation around topics, and Viermetz (2008), who calculated local actor centrality in content-based networks to find opinion leaders. To the best of our knowledge, content-based SNA has not been applied to mailing lists or to any kind of open source related data. 


\section{Data preparation}

The data were taken from the mailing lists Rhelp and R-devel from January 2008 to May 2009. The e-mails can be downloaded as compressed text files from the $\mathrm{R}$ website (https://stat.ethz. ch/pipermail/r-help/ and https://stat.ethz.ch/ pipermail/r-devel/). There is one text file for each month. The $\mathrm{R}$ code for the entire data preparation process can be downloaded from R-Forge https: //r-forge.r-project.org/projects/snatm/. First, the e-mails for each month were written into a single file using as.one. file () from the snatm package (example of the R-devel mailing list):

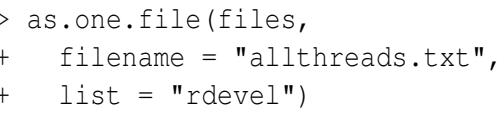

Then, the meta data (author, date, subject, thread-ID and e-mail-ID) and the e-mail content were extracted from these texts and transformed into a matrix.

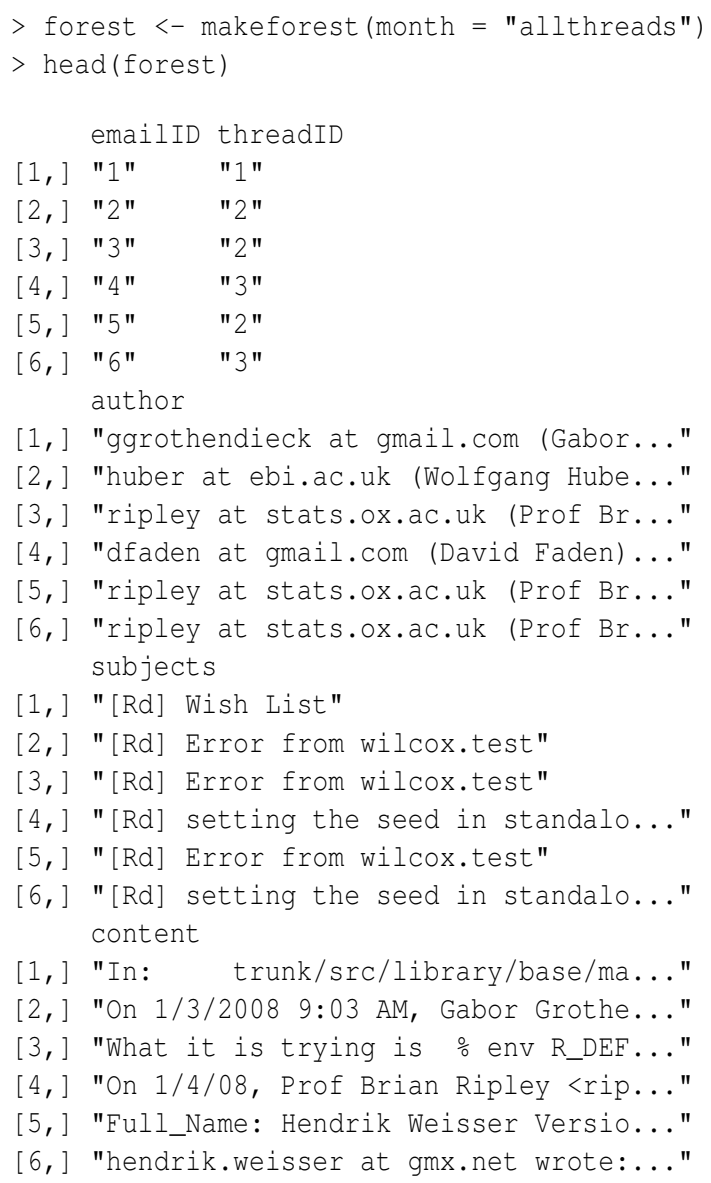

The function makeforest () from snatm is based on the tm.plugin.mail package (Feinerer et al., 2008) which uses the Message-IDs and In-Reply-To-IDs contained in the e-mail headers to assign a thread-ID to each e-mail (threads () from the tm.plugin.mail package). The e-mail-IDs sort the e-mails according to the sequence in which they were sent. Answers to e-mails that were sent before January 2008 had to be removed because in this case the threads () function cannot identify complete threads. Furthermore, citations and signatures were omitted from the e-mail content (removecitation() and removesignature () from the tm.plugin.mail package). The data contains 43760 R-help e-mails and 5008 R-devel e-mails.

\section{Data preparation for TM}

First, in order to reduce spelling ambiguities resulting from differences in American versus British English, the e-mail subjects and content were transformed into British English using the ae-to-be() function from the snatm package.

Second, the wn.replace() function from the snatm package was used to find groups of synonyms that were used in the texts based on the WordNet Database (Fellbaum, 1998; Wallace, 2007; Feinerer and Hornik, 2010). All terms in these groups of synonyms are replaced by one single representative of the group. However, in order to account for the R-specific vocabulary, not all of the synonym terms should be replaced. For example, the word "car" should not be replaced by "auto" where it "typically" refers to the car package. The wn.replace () function allows for manual deselection of certain terms, if they should not be replaced by a synonym.

Third, terms are stemmed using the Snowball stemmer stemDocument () from the tm package.

Fourth, term frequencies of the resulting terms (termFreq() from the tm package (Feinerer et al., 2008)) were calculated (separately for subjects and content). The function tolower() from the base package was applied and stopwords were ignored. Words of length less than three were omitted as the vast majority of them did not have a meaning in terms of abbreviations and the like, but were code chunks. Terms that were used less than 10 times (for subjects) or less than 20 times (for content) were ignored as well.

The four steps can be done all at once by the prepare.text () function from the snatm package.

\section{Data preparation for SNA}

To obtain a social network from the mailing list data, first, an alias detection procedure was performed on the Author-column of forest. It matched the different user names and e-mail addresses belonging to one and the same person by using the Levensthein Distance (agrep () (Levenshtein, 1966)) and a few transformations inspired by Bird et al. (2006) that can be found in find.aliases () from the snatm package. This way, the number of unique authors was reduced from 6393 to 5972 in R-help and from 1049 to 983 in R-devel.

Second, the new forest was transformed into an edge list (createedges () from snatm). Somebody answering an e-mail was connected to all the authors who wrote something before (chronologically) 
in the same thread as we assume that the respondent is aware of all the previous e-mails. After this, the edgelist was transformed into a matrix representation of a network (adjacency () from snatm).

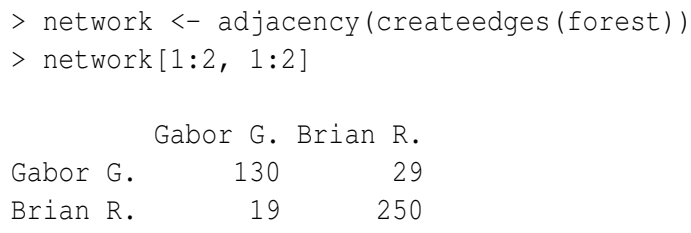

The resulting social network consists of nodes representing e-mail authors and directed valued lines indicating who answered whom and the number of mails sent in the corresponding direction. For example, Gabor Grothendieck answered on 29 of Brian Ripley's e-mails. If the diagonal is not zero the corresponding authors appeared several times in the same thread. (However, the diagonal will be ignored in the subsequent analysis.) We will call these networks "communication networks".

\section{Combining network data and textual data}

This section describes the crucial step where network data and textual data are combined. Three data preparation steps are needed.

\section{First step}

For all the subject terms that were used 10 times or more and all the content terms that appeared 20 times or more (using the prepared text corpora resulting from the prepare.text () function) a communication network was extracted that shows only the connections between authors who used this particular term. In a loop each term was assigned to subjectfilter, such that only connections between authors who used a certain term were extracted from forest.

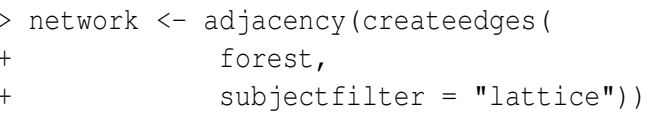

As an example, Figure 1 shows the communication network of all the R-help authors who used the term "lattice" in the e-mail subject.

Deepayan Sarkar, who is the author of the lattice package (Sarkar, 2008), is clearly the most central person in this network. This indicates that he answered nearly everyone having a question about lattice.

\section{Second step}

This step is based on the idea that someone's interest for a certain subject can be measured by his or her centrality or activity in the corresponding communication network. For example, we would conclude from Figure 1 that Deepayan Sarkar is very interested in the word "lattice". In SNA, there are several measures to calculate centrality, for example degree, closeness, betweenness and others. We chose to use the degree (number of direct neighbors; degree from the sna package (Butts, 2008)) because it measures activity while the others measure the connectedness to the entire network. The degree was calculated for all the nodes of networks that have more than 20 members. If a network has only 20 members or less, the results of centrality measures and thus the assumption of somebody being interested in a certain topic are considered not to be meaningful. Then, we created a network consisting of two sets of nodes (2-mode network), one representing e-mail authors and the other representing terms.

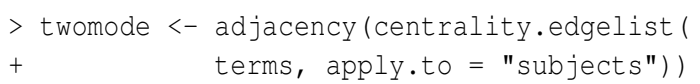

Each term and each author who used the term was connected by a line having the normalized degree centrality rank as line weight. (For instance the person with the highest degree in a certain communication network is connected to the corresponding word with a line weight of 1.) Thus, the line weight can be interpreted as the extent to which somebody is interested in a particular term in a [0,1] interval. Figure 2 shows the $0.45 \%$ strongest lines of R-help authors and subject-terms (largest connected subnetwork only). For example, Jim Lemon is connected to terms like "bar", "scatter", "axi(s)", "barplot" and "diagram", so he seems to be interested in plots.

\section{Third step}

In the third step, this 2-mode network was reduced to a 1-mode network by omitting the term nodes and connecting the author nodes that were adjacent to the term (shrink from the snatm package).

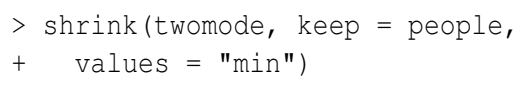

For example, in the 1-mode network John Fox and Gabor Grothendieck are connected because they are both connected to "mean" in the 2-mode network. The networks have line weights representing the minimum of the line weights two people were formally connected with through the term node. For example, in the 2-mode network John Fox was connected to the term "mean" with a line weight of 0.9957 and Gabor Grothendieck was also connected to "graph" but with a line weight of 1 . Then the two authors are connected with a line weight of 0.9957 in the 1-mode network, meaning that the extent to which they share interests is 0.9957 of 1 . We will call these networks "interest networks". 


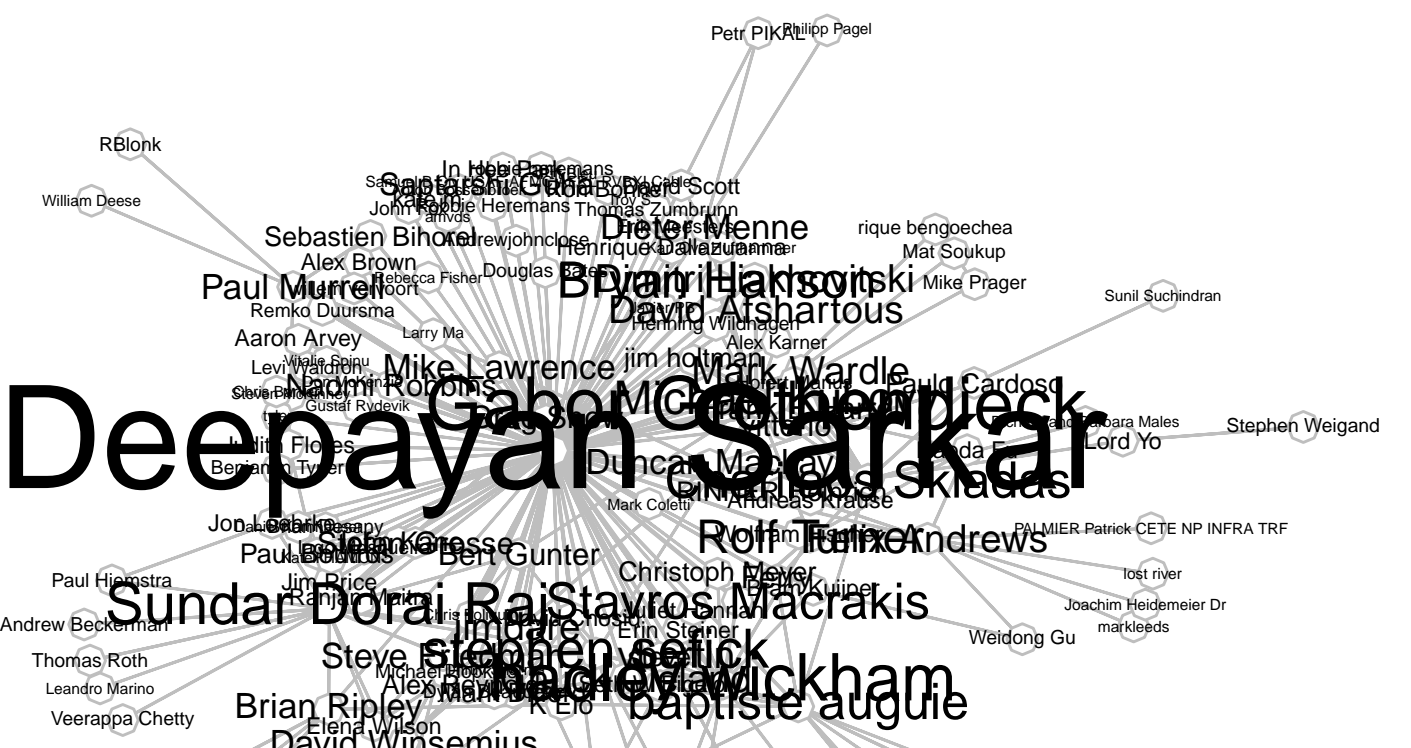

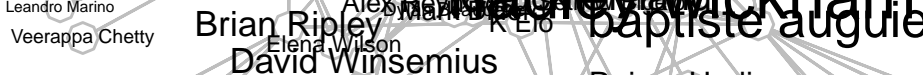

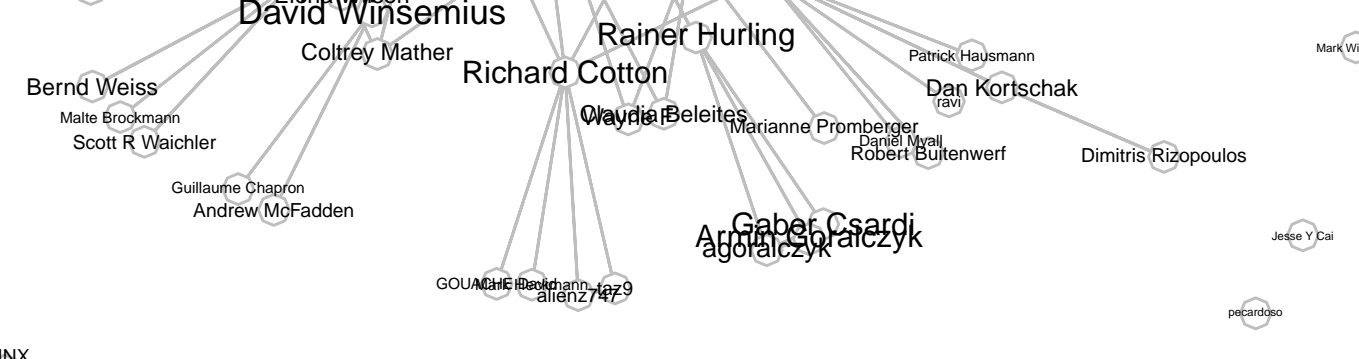

rraberetona

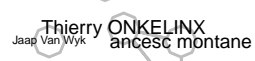

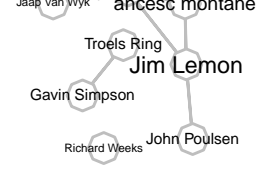

$$
\text { (n) }
$$
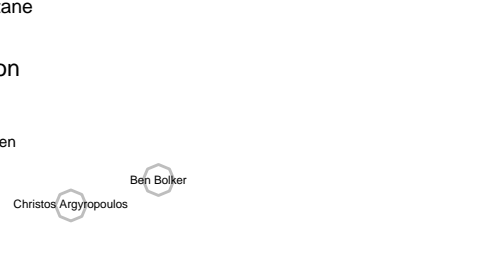


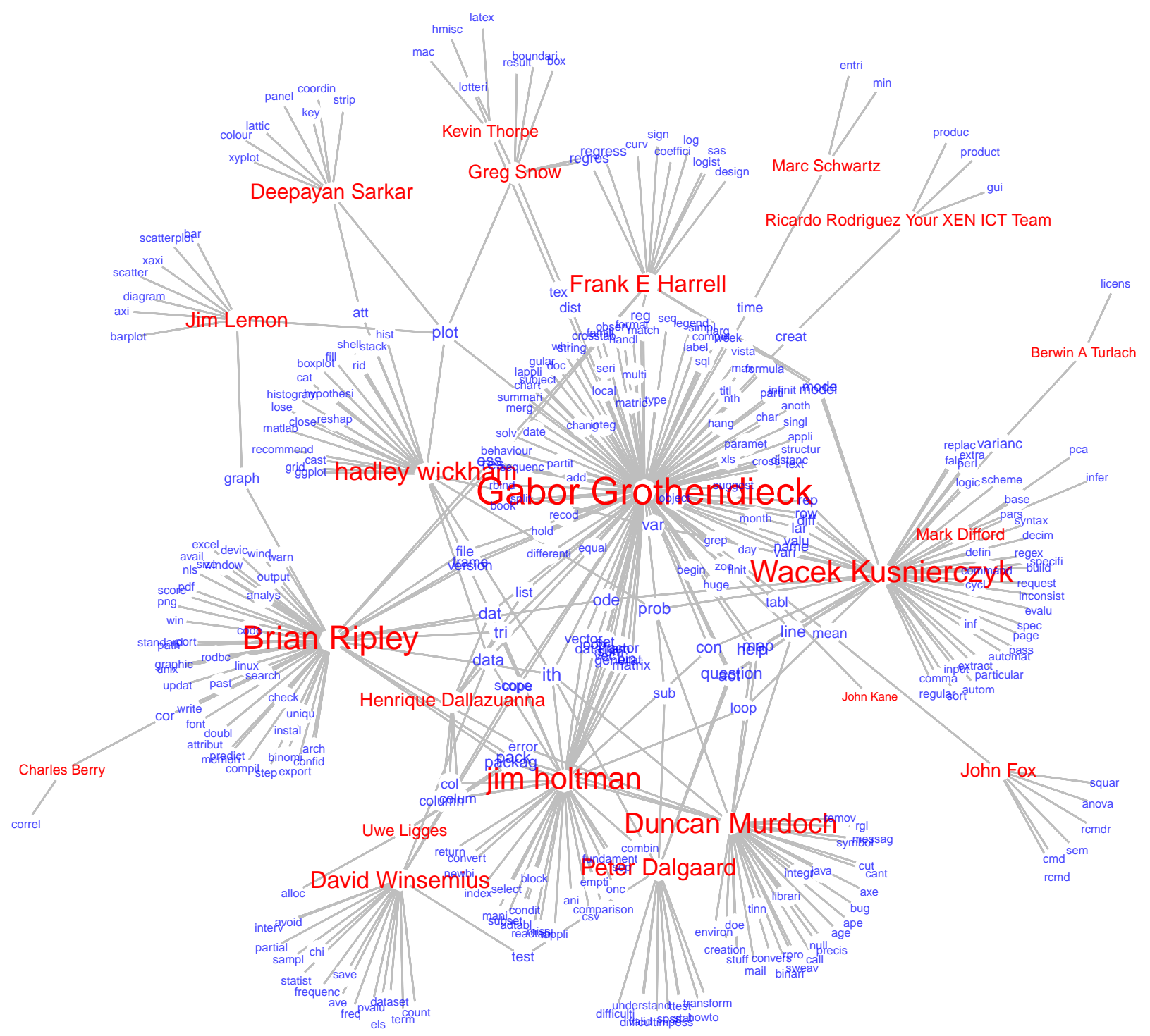

Figure 2: Author-term network showing the largest connected subnetwork of the $0.45 \%$ strongest connections between R-help authors and subject-terms. 


\section{Results: Comparison of communi- cation networks and interest net- works}

At this point, the two kinds of networks, the communication networks and the interest networks can be compared. The communication networks have line weights representing the number of e-mails exchanged. The line weights of the interest networks indicate the extent to which two people have similar interests in a $0-1$ interval. If we calculate the correlation between the line weights of both networks, we get the extent to which the fact of sharing interests is associated with communicating. We should expect that the more two people are interested in the same topic, the more e-mails they exchange.

There are two communication networks, one for R-help having 5972 nodes and one for R-devel having 983 nodes. Furthermore, there are four interest networks because the extent of shared interests was measured once by using the subjects and once by using the content for each of the mailing lists. The interest networks have fewer nodes because only the most frequently used terms were included into the interest analysis. Thus, people who only used less frequent terms do not appear in the interest networks. To compare the two kinds of networks the communication networks were reduced to those nodes who also appeared in the interest networks. Furthermore, the reduced communication network was permuted (permutation () from the snatm package) such that the sequence of author names assigned to rownames (network) is the same in both networks. However, the correlation between sharing interests and communicating is only approximately 0 for all possible combinations of communication networks and interest networks (Table 1). (The diagonals of the communication networks were set to 0 .)

\begin{tabular}{lrr}
\hline Interest & \multicolumn{2}{c}{ Communication network } \\
\cline { 2 - 3 } network & R-help & R-devel \\
\hline subjects & 0.030 & 0.070 \\
content & 0.009 & 0.044 \\
\hline
\end{tabular}

Table 1: Correlations between all combinations of communication networks and interest networks.

However, if the centrality of authors in the communication network is taken into account, we get a different picture. Figure 3 shows how the correlation between intensity of communication and extent of sharing interests changes ( $y$ axis) as the normalized centrality (degree, betweenness and closeness) of nodes increases ( $x$ axis). (Degree and closeness were calculated with the sna package (Butts, 2008), betweenness with the igraph package (Csardi and Nepusz, 2006).)
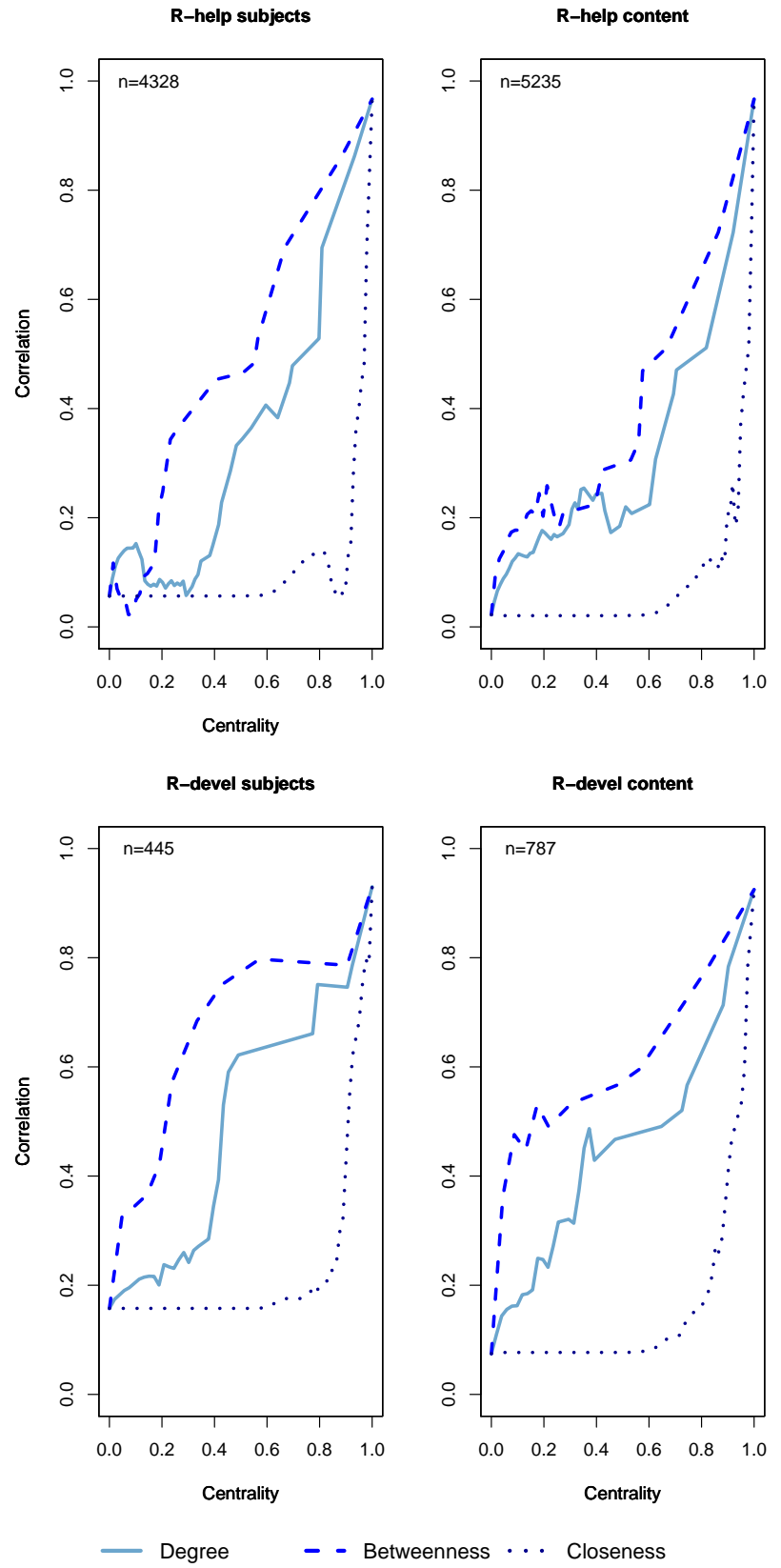

Figure 3: Correlation between intensity of communication and sharing interests (y-axis) and degree centrality of nodes (x-axis).

More precisely, it shows the correlations between communication networks and interest networks consisting of only those authors who have a higher or equally high centrality than indicated on the x-axis.

There is an almost linear increase in correlation in the R-help subjects network for the centrality measures degree and betweenness, whose distributions are highly right skewed. Closeness is approximately normally distributed. Thus, networks of people having an average closeness (around 0.5) are not correlated just as networks of authors with an average normalized degree (around 0). In the R-help 
content network, a relation between the centrality of authors and the correlation between sharing interests and communicating begins only at a centrality of around 0.5 for right skewed measures and around 0.95 for closeness. This is a result of the discussion topics sometimes changing in the course of threads. In the R-devel networks, the relation is also positive but not as stable, which is due to the much smaller network sizes $n$ of 445 (subjects) and 787 (content) compared to 4328 (subjects) and 5235 (content) in the R-help networks. The correlation in R-devel is generally higher than in R-help. In both, the R-help (for high centralities) and the R-devel networks, the lines are smoother when the subjects were used to find people's interests. This might be due to some additional chatting in the content while the subjects are more focused on the actual topics. However, the choice of subjects or content does not influence the general finding that sharing interests and communicating is the more associated the higher people's centrality scores are. This might be explained by impartiality or indifference of highly active authors towards the personality of their e-mail partners. They rather concentrate on the topics. Figure 4 supports this interpretation:
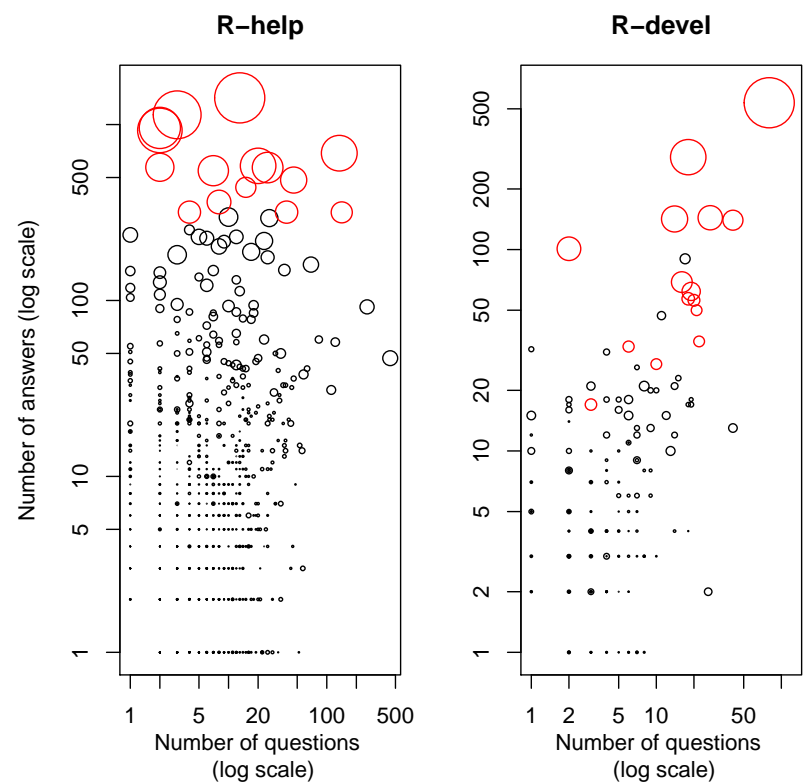

Figure 4: Scatterplot showing the number of questions (x-axis) and answers (y-axis) as well as the degree centrality (dot size) of each author.

Each point represents one author and shows how many questions (x-axis) and answers (y-axis) he or she wrote (ans.quest() from the snatm package). The larger a point the higher the author's degree centrality. The plots indicate that in R-help the 15 most active authors (red) write far more answers than questions. All but one author are developers of CRAN packages, which suggests that their motivation is to provide help concerning their packages or specific topics, no matter who asked the questions. In contrary, less central R-help authors are either more choosey with the choice of e-mail partners or they are not active enough to answer everyone having similar interests. In R-devel the proportion of answers to questions of central authors (red) is not different from less central authors. Still, Figure 3 suggests that there is a great correspondency between communicating and sharing interests among the most active authors. The low correlation between intensity of communication and extent of sharing interests for peripheral nodes in both mailing lists can be due to their very low activity which hinders the definition of their interests.

\section{Summary}

The paper showed how content-based SNA can be used to find people's interests in mailing list networks. By comparing communication graphs and networks showing who has similar interests, a relationship between the correlation of these two and node centrality could be found. Accordingly, the expected relationship between sharing interests and communicating exists only for very active authors while less active authors do not answer everyone who has similar interests. Thus, the communication efficiency can be regarded to be high for very active mailing list authors while it is moderate for mid-active authors. Additionally, the paper suggests using only the subjects to find the relationship between communicating and sharing interests because the content contains more noise.

\section{Code for figures 1 and 2}

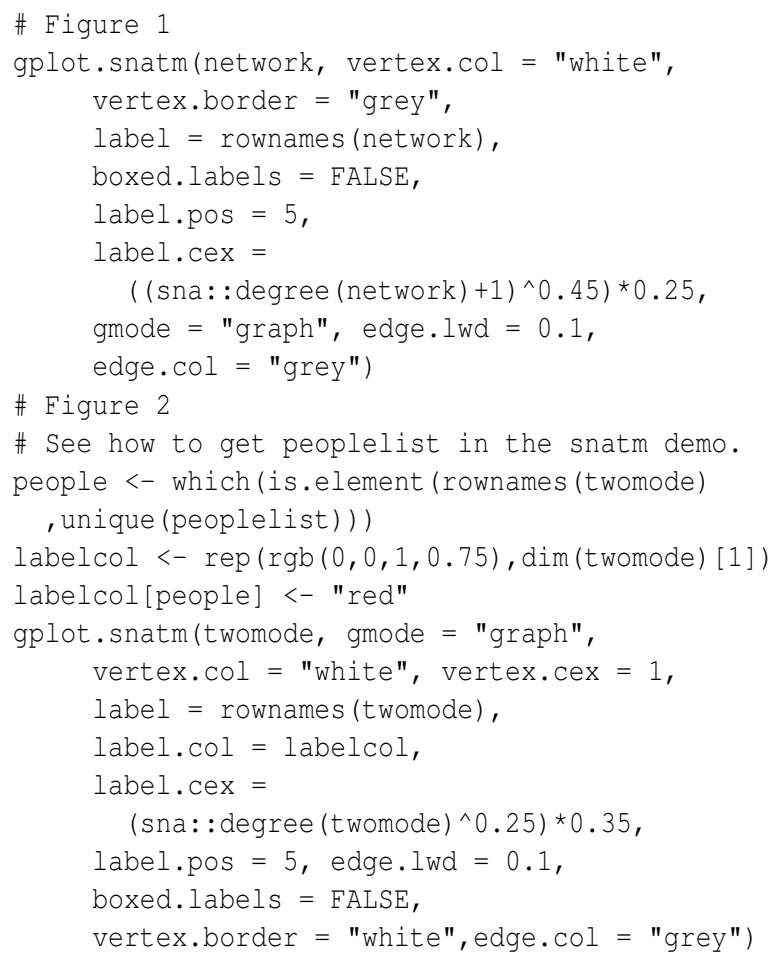




\section{Bibliography}

C. Bird, A. Gourley, P. Devanbu, M. Gertz, and A. Swaminathan. Mining email social networks. In Proceedings of the 2006 International Workshop on Mining Software Repositories, Shanghai, China, pages 137-143, 2006. URL http: / / macbeth. cs . ucdavis . edu/msr06.pdf.

S. Brin and L. Page. The anatomy of a largescale hypertextual web search engine. Computer Networks and ISDN Systems, 30(1-7):107117, 1998. URL http://wx.bsjh.tcc.edu.tw/ t2003013/wiki/images/8/8f/Anatomy.pdf.

C. T. Butts. Social network analysis with sna. Journal of Statistical Software, 24(6), 2008. URL http: //www. jstatsoft.org/v24/i06/.

J. Chang, J. B. Graber, and D. M. Blei. Connections between the lines: Augmenting social networks with text. In KDD '09: Proceedings of the 15th ACM SIGKDD international conference on Knowledge discovery and data mining, pages 169-178, New York, NY, USA, 2009. ACM. URL http: //www. cs . princeton.edu/ jbg/documents/kdd2009.pdf.

G. Csardi and T. Nepusz. The igraph software package for complex network research. InterJournal, Complex Systems:1695, 2006. URL http:// igraph.sf.net.

G. Erkan and D. R. Radev. Lexrank: Graphbased centrality as salience in text summarization. Journal of Artificial Intelligence Research, 2004. URL http: //tangra.si.umich.edu/ radev/ lexrank/lexrank.pdf.

I. Feinerer and K. Hornik. wordnet: WordNet Interface. $R$ package version 0.1-6., 2010. URL http: //CRAN.R-project.org/package=wordnet.

I. Feinerer, K. Hornik, and D. Meyer. Text mining infrastructure in R. Journal of Statistical Software, 25(5):1-54, 2008. ISSN 1548-7660. URL http: //www. jstatsoft.org/v25/i05.

C. Fellbaum. WordNet: An Electronic Lexical Database. Bradford Books, 1998.

J. M. Kleinberg. Authoritative sources in a hyperlinked environment. Journal of the ACM, 46(5): 604-632, 1999. URL http://www.cs. cornell.edu/ home/kleinber/auth.pdf.

V. I. Levenshtein. Binary codes capable of correcting deletions, insertions, and reversals. Technical Report 8, Soviet Physics Doklady, 1966.
A. McCallum, X. Wang, and A. Corrada-Emmanuel. Topic and role discovery in social networks with experiments on Enron and academic email. Journal of Artificial Intelligence Research, 30:249-272, 2007. URL http://www. cs. umass. edu/ mccallum/ papers/art-ijcai05.pdf.

D. Sarkar. Lattice: Multivariate Data Visualization with R. Springer, 2008.

L. Vanderwende, M. Banko, and A. Menezes. Event-centric summary generation. In In Working Notes of DUC 2004, 2004. URL http://www-nlpir.nist.gov/projects/duc/ pubs/2004papers/microsoft. banko.pdf.

P. Velardi, R. Navigli, A. Cucchiarelli, and F. D'Antonio. A new content-based model for social network analysis. In Proceedings of the 2008 IEEE International Conference on Semantic Computing, pages 18-25, Washington, DC, USA, 2008. IEEE Computer Society. URL http://www. dsi. uniromal. it/ velardi/ICSCpubblicato.pdf.

M. Viermetz. Partitioning Massive Graphs for Content Oriented Social Network Analysis. PhD thesis, Heinrich-Heine-Universität Düsseldorf, June 2008. URL http://docserv.uni-duesseldorf. de/servlets/DerivateServlet/Derivate-8825/ diss_viermetz_pdfalb.pdf.

M. Wallace. Jawbone Java WordNet API, 2007. URL http://mfwallace.googlepages.com/jawbone. html.

Angela Bohn, Kurt Hornik, Patrick Mair Institute for Statistics and Mathematics Department of Finance, Accounting and Statistics Wirtschaftsuniversität Wien

Augasse 2-6

A-1090 Wien

Austria

angela.bohn@gmail.com

kurt.hornik@wu-wien.ac.at

patrick.mair@wu.ac.at

\section{Ingo Feinerer}

Database and Artificial Intelligence Group

Institute of Information Systems

Technische Universität Wien

Favoritenstrasse 9

A-1040 Wien

Austria

feinereralogic.at 